\title{
A Non-classical Presentation of Schmidt's Syndrome: A Rare and a Lethal Disease
}

\author{
Suman Preet Kaur Bhullar, ${ }^{\mathrm{a}, \mathrm{b}}$, Raouf Seifeldina, Nikhil Hemady ${ }^{\mathrm{a}}$
}

\begin{abstract}
Schmidt's syndrome is a rare autoimmune endocrine disease in which the patient suffers from primary addison's disease and primary hypothyroidism. Currently, Schmidt's Syndrome has been classified as a part of the inheritable Polyglandular Autoimmune Syndromes (PAS). It is more common in females and has a complex inheritance pattern when familial (50\%). Though it is treatable if diagnosed early, but many patients die within one year of being diagnosed. A 25 year-old male with a known history of adrenal insufficiency, hypothyroidism, and hypopituitarism presented with weakness, confusion and fainting spells since 3 weeks. A physical examination revealed vitiligo on the digits. Laboratory investigations showed elevated TSH, decreased testosterone and decreased ACTH, all consistent with a diagnosis of Schmidt's syndrome. He was started on appropriate hormone replacement therapy Adrenal insufficiency was treated earlier and followed by treatment of thyroid insufficiency. The patient improved and is on a regular follow up. We report a unique case as our patient is a young male, who rarely present with Schmidt's syndrome. He did not have Type I diabetes, but developed Growth Hormone (GH) deficiency. Moreover PAS Type II is often inherited in an autosomal dominant pattern, while in our patient there was no family history of PAS. There are very few case reports in literature of atypical presentations of Schmidt's syndrome which can add to the literature to help in earlier diagnosis and better outcomes of such patients.

To conclude, a high index of suspicion is needed for an early diagnosis and appropriate hormonal replacement therapy of Schmidt's syndrome, which is a rare disease, but is a life threatening condition if not diagnosed and treated early.
\end{abstract}

Keywords: Polyglandular; Autoimmune; Schmidt's; Addison's;

\footnotetext{
Manuscript accepted for publication June 12, 2013

${ }^{a}$ Department of Family Medicine, Doctor's Hospital of Michigan, Pontiac, MI, USA

${ }^{\mathrm{b}}$ Corresponding author: Suman Preet Kaur Bhullar, Department of Family Medicine, Doctor's Hospital of Michigan, 461, W Huron Street, Pontiac, Michigan, USA. Email: drsbhullar@gmail.com
}

doi: http://dx.doi.org/10.4021/jem174w
Hypothyroidism

\section{Introduction}

Schmidt's syndrome is a rare $(1.4-4.5 / 100,000)$, autoimmune endocrine disease in which the patient suffers from primary Addison's disease and primary hypothyroidism [1]. Currently, Schmidt's syndrome has been classified as a part of the inheritable Polyglandular Autoimmune Syndromes (PAS) [2].

Polyglandular auto-immune syndromes are rare entity. They are characterized by immune dysfunction affecting two or more endocrine glands as well as certain non-endocrine organs. The PAS syndromes are classified as two main types: PAS Type I and PAS Type II. PAS Type II is also known as Schmidt's syndrome.Type I polyglandular autoimmune syndrome (PAS Type I) consists of Addison's disease, hyperparathyroidism and chronic mucocutaneous candidiasis. Type II polyglandular autoimmune syndrome (PGA Type II) consists of Addison's disease, autoimmune thyroid disease and Type I diabetes, grave's disease, autoimmune thyroiditis, and can be associated with other autoimmune disorders like myasthenia gravis, primary hypogonadism, vitiligo, alopecia and serositis [1].

Pernicious anemia also occurs with increased frequency in patients with this syndrome.

The pattern of inheritance in Schmidt's syndrome is autosomal dominance, it is more frequently encountered in women, and the male-to-female ratio is 1:3 [2]. It is treatable if diagnosed early, but many patients die within one year of being diagnosed [3]. Diagnosing such cases could be challenging and mislead early recognition and treatment of such cases can be life saving.

We report a case in which a patient had primary adrenal insufficiency, autoimmune hypothyroidism and gonadal failure and was diagnosed as "Schmidt's syndrome" (PGA Type II). This patient did not have Type I diabetes, but he developed Growth Hormone (GH) deficiency. It could be attributed to autoimmune hypophysitis, which is a very rare component of the syndrome. Moreover PAS Type II is often 
inherited in an autosomal dominant pattern, in our patient there has been no known family history of PAS.

\section{Case Report}

A 25 year-old African American male with a history of adrenal insufficiency, hypothyroidism, and hypopituitarism was admitted with weakness and weight loss. He also had complaints of confusion, decreased ability to perform normal daily living activities with sporadic "fainting" spells since few weeks. There were no complaints of fever, seizures and focal neurological deficit. Also there was no history of hair loss, joint pain and other features of autoimmune disease.

Physical examination revealed, no alopecia, BMI of $17.5 \mathrm{~kg} / \mathrm{mt}^{2}$, pulse rate of $56 / \mathrm{min}$, and Blood pressure of $-122 / 71 \mathrm{mmHg}$. Rest of the examination was normal except for vitiligo on digits and a flat affect. CNS and other systemic examinations were normal.

Initial laboratory sttudies including $\mathrm{CBC}$, blood sugar, renal function tests, liver function tests, serum electrolytes and fasting lipid profiles were within normal limits. But the total T3 $23 \mathrm{ng} / \mathrm{dl}$, Free T4 $<0.20 \mathrm{ng} / \mathrm{dl}$, TSH $58.18 \mathrm{uIU} / \mathrm{ml}$, Testosterone levels 226, ACTH levels of less than 2 was suggestive of primary adrenal insufficiency.

Based on the history and laboratory findings, the patient was diagnosed to have primary adrenal insufficiency, autoimmune hypothyroidism and hypogonadism. Diagnosis of Polyendocrine syndrome Type II (Schmidt's syndrome) had been made and confirmed at University of Michigan. He was started on Synthroid $225 \mathrm{mcg}$ daily, Florinef $0.1 \mathrm{mg}$ once daily, Cortef $10 \mathrm{mg} / 7.5 \mathrm{mg} / 5 \mathrm{mg}$ at morning/noon/evening, Testosterone $150-200 \mathrm{mg}$ injection every 14 to 21 days, Human growth hormone $0.3 \mathrm{mg}$ once daily. He improved symptomatically with the treatment over five days and at the time of his discharge the patient's complaints of confusion, dizziness and weakness had improved. During his follow-up after one month he was completely normal and is doing well on regular follow up.

\section{Discussion}

Polyglandular autoimmune syndromes (PAS) are a heterogeneous group of diseases in which a genetically caused dysfunction of the immune system leads to a destruction of endocrine glands with subsequent loss of function [3]. In addition non-endocrine autoimmune diseases are also frequently present. The polyglandular autoimmune syndrome (PAS) is defined as the manifestation of at least two endocrine autoimmune diseases [1]. In order to take the wide spectrum of components and the variations of the disease fully into account, PAS is usually divided up into the rare juvenile type (PAS Type I) and the more common adult type (PAS Type
II - IV) [4]. PAS Type III comprises of autoimmune thyroid diseases associated with other autoimmune diseases (Type I diabetes, atrophic gastritis, pernicious anemia, vitiligo, alopecia, myasthenia gravis), excluding Addison's disease and/ or hypoparathyroidism [3].

PAS Type I, also known as APECED (autoimmune polyendocrinopathy-candidiasis-ectodermal dystrophy), MEDAC (multiple endocrine deficiency autoimmune candidiasis syndrome), juvenile autoimmune polyendocrinopathy, or Whitaker's syndrome (OMIM 240300), is classically defined by the association of at least two of three major component diseases: chronic mucocutaneous candidiasis, primary hypoparathyroidism, and autoimmune adrenal insufficiency [5]. In most cases PAS Type I appears in infancy and childhood.

Polyglandular autoimmune syndrome Type II is also known as Schmidt's syndrome. Polyglandular autoimmune syndrome Type II (PAS Type II) is the most common of the immunoendocrinopathy syndromes. It is characterized by the obligatory occurrence of autoimmune Addison's disease in combination with thyroid autoimmune diseases and/or Type I diabetes mellitus (also known as insulin-dependent diabetes mellitus, or IDDM) [3]. Primary hypogonadism, myasthenia gravis, and celiac disease also are commonly observed in this syndrome [6]. Moreover, the combined occurrence of Addison's disease and chronic lymphocytic thyroiditis was first reported in two patients by Schmidt in 1926 [7].

According to recent epidemiological data, the frequency of PAS Type II is estimated to be 4 - 5 cases per 100,000 individuals in the general population [1]. The pattern of inheritance is autosomal dominance, it is more frequently encountered in women, and the male-to-female ratio is $1: 3$ [2]. This syndrome has a peak incidence at ages 20 - 60 years, mostly in the third or fourth decade, and it is common for multiple generations to be affected by one or more component diseases. There is familial clustering and family members of patients are often affected [8].

Thyroid disease is the most common glandular disorder to accompany adrenal insufficiency in individuals with Schmidt's syndrome. Thyroid disease, as a part of Schmidt's syndrome can occur in the form of chronic autoimmune thyroiditis (Hashimoto's thyroiditis) or Graves' disease. Hashimoto's is much more common and occurs in 95 to 97 percent of patients with Schmidt's syndrome who develop a thyroid disease [3]. Grave's disease occurs in 3 to 5 percent of patients who develop thyroid disorders. Adrenal insufficiency is the initial manifestation in about $50 \%$ of patients, occurring simultaneously with diabetes mellitus and autoimmune thyroid disease in about $30 \%$ and $20 \%$ respectively [9]. About 10 percent of patients with Schmidt's syndrome also develop vitiligo.

As with most autoimmune disorders, the predominant known genetic determinant of susceptibility to PAS Type II resides in the human leukocyte antigens (HLA) region (i.e., major histocompatibility complex). The class II HLA 
haplotypes DR3 (DQB*0201) and DR4 (DQB1*0302) are strongly linked with component disorders of this syndrome. It is highly likely that there is a complex interaction between non-HLA loci and environmental factors [9].

Many of the endocrine disorders of PAS are adequately treated with hormonal replacement therapy if the disease is recognized early. The therapies regarding the different components of Type II PAS are similar whether they occur as single or in multiple associations with other autoimmune diseases.

Diagnosing of Schimdt's syndrome can be challenging and misleading too. Early recognition and treatment of such cases can stand between the life and death of a patient.

Our reported case is unique, as our patient is a young male, who rarely present with Schmidt's syndrome and is a non classical presentation. In our case, patient did not have Type I diabetes, but he developed GH deficiency. It could be attributed to autoimmune hypophysitis, which is a very rare component of the syndrome. Although PAS Type II is often inherited in an autosomal dominant pattern, in our patient there has been no known family history of PAS.

Adrenal insufficiency and autoimmune thyroiditis were the presenting endocrinopathies as well as presumptive autoimmune hypophysitis. This combination constitutes rare manifestations of the syndrome, especially at a young age. Very few cases of this syndrome have been reported in males.

Every patient with idiopathic endocrine deficiency should be screened for insufficiencies in other endocrine organs. A high index of suspicion is needed for an early diagnosis and hormonal replacement therapy of Schmidt's syndrome, which is a rare disease, but is a life threatening condition if not diagnosed and treated early.

\section{Conclusions}

Though Schimdt's syndrome is a rare disease, but can be a life threatening condition if not diagnosed and treated early. Atypical presentations of such a condition are possible and it is recommended that every patient with idiopathic endocrine deficiency should be screened for insufficiencies of other endocrine organs. A high index of suspicion is needed for an early diagnosis and appropriate hormonal replacement therapy for Schmidt's syndrome.

\section{Disclosure}

All the authors have nothing to disclose.

\section{References}

1. Laureti S, Vecchi L, Santeusanio F, Falorni A. Is the prevalence of Addison's disease underestimated? J Clin Endocrinol Metab. 1999;84(5):1762.

2. Ten S, New M, Maclaren N. Clinical review 130: Addison's disease 2001. J Clin Endocrinol Metab. 2001;86(7):2909-2922.

3. Maurer A, Schwarting A, Kahaly GJ. [Polyglandular autoimmune syndromes]. Z Rheumatol. 2011;70(9):752754, 756-759.

4. Hansen MP, Kahaly GJ. [Autoimmune polyglandular syndromes]. Dtsch Med Wochenschr. 2013;138(7):319326; quiz 327-318.

5. Weiler FG, Dias-da-Silva MR, Lazaretti-Castro M. Autoimmune polyendocrine syndrome type 1: case report and review of literature. Arq Bras Endocrinol Metabol. 2012;56(1):54-66.

6. Gupta AN, Nagri SK. Schmidt's syndrome - Case report. Australas Med J. 2012;5(6):292-295.

7. Schmidt MB. Eine biglandulare Erkrankung (Nebennieren und Schilddrüse) bei Morbus Addisonii. Verh Dtsch Ges Pathol. 1926;21:212-221.

8. Neufeld M, Maclaren N, Blizzard R. Autoimmune polyglandular syndromes. Pediatr Ann. 1980;9(4):154-162.

9. Graves L, 3rd, Klein RM, Walling AD. Addisonian crisis precipitated by thyroxine therapy: a complication of type 2 autoimmune polyglandular syndrome. South Med J. 2003;96(8):824-827. 